\title{
Analysis of Risk Factors of Acute Myocardial Infarction Combined with Ventricular Septal Rupture
}

\section{Zhang $\mathrm{Y}^{*}$, Chen Y, Liu J, Li M, Liu Y, Gao C and Wang Z}

Henan University People’s Hospital, Fuwai Huazhong Cardiovascular Hospital, China

*Corresponding author: Wang Z, Henan University People’s Hospital, Fuwai Huazhong Cardiovascular Hospital, China, Tel: +8515515909078, E-mail: zhang04212@163.com

Citation: Zhang Y, Chen Y, Liu J, Li M, Liu Y, et al. (2021) Analysis of Risk Factors of Acute Myocardial Infarction Combined with Ventricular Septal Rupture. J Clin Trials Exp Stud 1(1): 101

Received Date: December 17, 2021 Accepted Date: December 28, 2021 Published Date: December 30, 2021

\begin{abstract}
Objective: To analyze the clinical characteristics and risk factors of ventricular septal rupture (VSR) after acute myocardial infarction (AMI).

Methods: A total of 2090 cases of acute myocardial infarction and 63 cases of acute myocardial infarction combined with ventricular septal perforation were collected from Fuwai Huazhong Cardiovascular Hospital from September 2017 to January 2021. 189 cases of type 1 acute myocardial infarction were randomly selected according to the ratio of 1:3 as a control group, the patients collected and analyzed clinical data, the risk factors of AMI and VSR.

Results: Compared with the control group, the age, female, serum creatinine, serum potassium, serum magnesium, N-terminal B-type natriuretic peptide (NT-proBNP), LDH1, hemoglobin, total protein, and albumin were higher in the ventricular septal rupture group, Serum chloride ion, and low blood pressure at admission, the difference was statistically significant $(\mathrm{P}<0.01$ or 0.05$)$. The results of multivariate logistic regression analysis showed that glycosylated hemoglobin $(\mathrm{OR}=1.66,95 \% \mathrm{CI} 1.04-2.95, \mathrm{p}=0.03)$ was related to the mortality of ventricular septal rupture after myocardial infarction; age $>60$ years, gender, diseased blood vessels, albumin $<35 \mathrm{~g} / \mathrm{L}, \mathrm{SBP}<90 \mathrm{mmHg}, \mathrm{LVEF}<40 \%, \mathrm{LVESD}$ is related to ventricular septal rupture after acute myocardial infarction (all $\mathrm{p}<0.01$ or 0.05 ).
\end{abstract}

Conclusion: elderly, female, left anterior descending artery disease, hypotension, low protein Patients with acute myocardial infarction with hyperemia, hypochloremia, and left ventricular systolic dysfunction are prone to ventricular septal rupture.

Keywords: Acute Myocardial Infarction; Ventricular Septal Rupture; Risk Factors; Interventional Therapy 


\section{Introduction}

Acute myocardial infarction (AMI) combined with ventricular septal rupture (VSR) is a rare but life-threatening complication. Before the era of thrombolytic therapy, the incidence of VSR was 1-2\%, and the survival rate after VSR was extremely poor. The hospital mortality rate for patients undergoing surgery was $45 \%$, and the hospital mortality rate for patients receiving drug treatment was $90 \%{ }^{[1]}$. With the advent of the era of thrombolysis, the incidence of ventricular septal rupture after acute myocardial infarction has dropped significantly, only $0.17-0.31 \%$ [1,2]. The emergence of emergency reperfusion strategies (including thrombolysis and PCI) makes VSR Patients benefit from [3,4]. Unfortunately, despite the decrease in incidence, the mortality (41-80\%) and prognosis of such patients are still poor $[1,5,6]$. Studies have confirmed that elderly women, anterior myocardial infarction without collateral circulation are risk factors for ventricular septal rupture after acute myocardial infarction [7,8]. Basic research shows that matrix metalloproteinases and inflammatory cytokines play an important role in patients with ventricular septal rupture after myocardial infarction $[9,10]$. Therefore, it is very important to identify and treat the perforation of the ventricular septum as soon as possible after acute myocardial infarction, which can significantly improve the prognosis of patients [11].

As for the mechanism of myocardial infarction combined with ventricular septal rupture, it is not yet clear. Therefore, the purpose of this study is to: (1) compare the clinical and angiographic characteristics of patients with VSR after AMI and determine the risk factors of patients with VSR after AMI; (2) The evaluation includes the effect of the treatment plan for patients with VSR after AMI, and determines the related risk factors after VSR after AMI and the related factors that affect the prognosis.

\section{Methods}

\section{Research objects and groups}

A total of 2090 cases of acute myocardial infarction and 63 cases of acute myocardial infarction combined with ventricular septal perforation (VSR group) were collected from Fuwai Huazhong Cardiovascular Hospital from September 2017 to January 2021. 1189 cases of type 1 acute myocardial infarction were randomly selected according to the ratio of 1:3 as a control group (non-VSR group). Collect information about patient demographics,clinical characteristics,preoperative clinical conditions, echocardiographic characteristics, surgical data and treatment status through the electronic medical record. We only included post-infarction ventricular septal rupture in this study.

The diagnostic criteria for acute myocardial infarction is that cardiac biomarkers exceed the upper limit of $99 \%$ of the reference value, accompanied by at least one of the following criteria: (1) myocardial ischemic symptoms; (2) new ST-T changes or new left bundle branch Conduction block; (3) Pathological Q waves appear on the electrocardiogram; (4) Newly appearing loss of viable myocardium, or new imaging evidence of segmental ventricular wall motion abnormalities; (5) Thrombosis in coronary arteries found by angiography [12]. The definition of type 1 myocardial infarction: MI caused by atherosclerotic thrombotic coronary artery disease, detects the increase and/or decrease of troponin (cTn), at least one of which is higher than the 99\% reference value online, and has one of the diagnostic criteria for acute myocardial infarction. VSR diagnostic criteria: (1) In clinical examination, a new loud and rough total systolic murmur appeared in the 4 to 5 intercostal spaces on the left edge of the sternum; (2) Transthoracic echocardiography showed ventricular septal detachment and color Doppler was used to show the left direction Right shunt; (3) Left ventricular angiography in the left anterior italic position revealed that the contrast agent shunted from the left ventricle to the right ventricle; (4) Placement of the pulmonary artery catheter showed a sharp increase in mixed venous oxygen saturation [13].

Exclusion criteria: (1) Other types of myocardial infarction; (2) Sudden cardiac arrest or pulseless electrical activity may have ventricular septal defect; (3) Infective endocarditis, neoplasms in the heart, or other infections (4) Combined with hemorrhagic disease and thrombocytopenia; (5) Combined with obvious liver and kidney dysfunction; (6) Heart insufficiency, intolerance of surgery; (7) Severe valvular disease requires surgical treatment; (8) The survival period of other malignant diseases is less than 6 months. 


\section{Treatment methods}

Coronary angiography uses the standard percutaneous radial artery approach. In the case of unstable hemodynamics, cardiac mechanical circulatory assistance (MCS) is required; the femoral artery approach is used. If the hemodynamics continues to deteriorate after the use of MCS, percutaneous closure is performed immediately. Primary PCI should be done immediately to all cases, but the timing of PCI and antiplatelet therapy is determined by the doctor based on the patient's condition.

\section{Statistical analysis}

SPSS 21.0 was used for data analysis, the measurement data conforming to the normal distribution were expressed by the mean standard deviation (S), and the t-test of independent samples was used for the comparison between groups; the measurement data of the skewed distribution was expressed by the interquartile range M (Q1, Q3), non-parametric test was used for statistical analysis of differences between groups. The count data was descriptive statistics by the number of cases and the constituent ratio (rate), and the chi-square test was used for comparison between groups. Patients who died one year after surgery and whether ventricular septal rupture occurred were two groups of dependent variables, and statistically significant indicators were included in univariate analysis. Multivariate logistic regression was used to analyze the risk factors of death and ventricular septal rupture in VSR patients at one year. Take $=0.05$ as the test level, $\mathrm{P}<0.05$ as the difference is statistically significant (Figure 1 ).

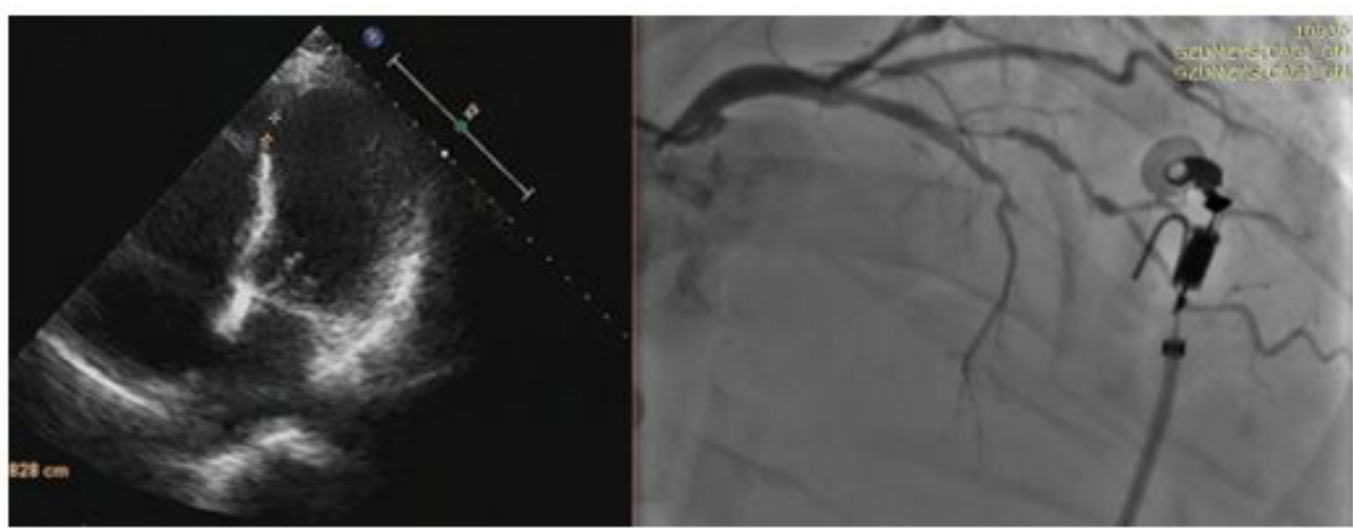

Figure 1: Ventricular septal rupture occurred were two groups of dependent variables

\section{Result}

1. The baseline data comparison is shown in Table 1: Compared with the control group, the patients in the ventricular septal rupture group are older, and the proportion of men and the history of smoking and drinking is lower, and the differences are statistically significant.

\begin{tabular}{|c|c|c|c|}
\hline Variable & $\begin{array}{c}\text { VSR group } \\
(\mathbf{n = 6 3 )}\end{array}$ & $\begin{array}{c}\text { non-VSR group } \\
(\mathbf{n = 1 8 9})\end{array}$ & P value \\
\hline Age [years, M (Q1,Q3)] & $70(66,74)$ & $61(51.5,70)$ & $<0.01$ \\
\hline Female sex & $34(54 \%)$ & $32(16.9 \%)$ & $<0.01$ \\
\hline Smoking & $25(39.7 \%)$ & $123(65.1 \%)$ & $<0.01$ \\
\hline Drinking & $17(27 \%)$ & $88(46.6 \%)$ & 0.006 \\
\hline
\end{tabular}

Table 1: Baseline data of patients 
2. The medical history data is shown in Table 2: Compared with the control group, the diseased blood vessels in the VSR group mostly occurred in $\operatorname{LAD}(73 \%)$, with a $\mathrm{P}$ value of $<0.01$ or 0.05 , and the difference was statistically significant.

\begin{tabular}{|l|c|c|c|}
\hline \multicolumn{1}{|c|}{ Variable } & $\begin{array}{c}\text { VSR group } \\
(\mathbf{n = 6 3 )}\end{array}$ & $\begin{array}{c}\text { non-VSR group } \\
(\mathbf{n = 1 8 9 )}\end{array}$ & P value \\
\hline Hypertension & $35(55.6 \%)$ & $95(50.3 \%)$ & 0.47 \\
\hline Diabetes mellitus & $20(31.7 \%)$ & $55(29.1 \%)$ & 0.69 \\
\hline Coronary artery disease LM & & & \\
\hline LAD & 0 & $17(9 \%)$ & \\
\hline LCX & $46(73 \%)$ & $70(37.1 \%)$ & 0.002 \\
\hline RCA & $2(3.2 \%)$ & $29(15.3 \%)$ & \\
\hline & $11(17.5 \%)$ & $73(38.6 \%)$ & \\
\hline
\end{tabular}

Note: LM (left main coronary artery): left main artery; LAD (left anterior descending coronary artery): left anterior descending coronary artery; LCX (left circumflex coronary artery): left circumflex coronary artery; RCA (right coronary artery): Right coronary artery. AMI (acute myocardial infarction): acute myocardial infarction; VSR (ventricular septal rupture): ventricular septal rupture.

Table 2: Medical history data

3. Comparison of laboratory test results is shown in Table 3: Compared with the control group, the levels of hemoglobin, albumin, and serum chloride in the ventricular septal rupture group were lower at admission, and the levels of serum creatinine and serum potassium were higher $(\mathrm{P}<0.01$ Or 0.05$)$, the other test indicators were compared between the two groups, and the difference was not statistically significant.

\begin{tabular}{|c|c|c|c|}
\hline Variable & $\begin{array}{c}\text { VSR group } \\
(\mathbf{n}=\mathbf{6 3})\end{array}$ & $\begin{array}{c}\text { non-VSR group } \\
(\mathbf{n}=\mathbf{1 8 9})\end{array}$ & $\boldsymbol{P}$ value \\
\hline Hemoglobin $(\mathrm{g} / \mathrm{L}, \bar{X} \pm \mathrm{S})$ & $121.75 \pm 18.23$ & $132.21 \pm 17.28$ & $<0.01$ \\
\hline $\mathrm{TP}(\mathrm{g} / \mathrm{L}, \bar{X} \pm \mathrm{S})$ & $62.97 \pm 6.18$ & $64.24+5.60$ & 0.13 \\
\hline Albumin $[\mathrm{g} / \mathrm{L}, \mathrm{M}(\mathrm{Q} 1, \mathrm{Q} 3)]$ & $35.90(33.80,38.90)$ & $39.40(37.05,41.9)$ & $<0.01$ \\
\hline Creatinine $[\mu \mathrm{mol} / \mathrm{L}, \mathrm{M}(\mathrm{Q} 1, \mathrm{Q} 3)]$ & $88.00(67.00,109.00)$ & $73.00(63.50,85.00)$ & $<0.01$ \\
\hline $\mathrm{HbA1c}[\%, \mathrm{M}(\mathrm{Q} 1, \mathrm{Q} 3)]$ & $6.41(5.79,8.56)$ & $6.03(5.61,7.43)$ & 0.11 \\
\hline $\mathrm{K}+[\mathrm{mmol} / \mathrm{L}, \mathrm{M}(\mathrm{Q} 1, \mathrm{Q} 3)]$ & $4.44(4.08,4.90)$ & $4.13(3.84,4.47)$ & 0.001 \\
\hline $\mathrm{Mg} 2+[\mathrm{mmol} / \mathrm{L}, \mathrm{M}(\mathrm{Q} 1, \mathrm{Q} 3)]$ & $0.96(0.86,1.04)$ & $0.93(0.87,0.99)$ & 0.21 \\
\hline $\mathrm{Cl}-[\mathrm{mmol} / \mathrm{L}, \mathrm{M}(\mathrm{Q} 1, \mathrm{Q} 3)]$ & $99.00(95.00,102.00)$ & $102.00(99.00,104.00)$ & $<0.01$ \\
\hline
\end{tabular}

Table 3: Laboratory inspection

4. Comparison of myocardial infarction-related examination results is shown in Table 4: Compared with the control group, patients in the ventricular septal rupture group had lower levels of systolic and diastolic blood pressure, left ventricular ejection fraction, and left ventricular end-systolic diameter and NT- The levels of proBNP and LDH1 were higher $(\mathrm{P}<0.01$ or 0.05$)$. There were 14 cases in the ventricular septal rupture group with left ventricular ejection fraction less than $45 \%$, accounting for $22 \%$. 


\begin{tabular}{|c|c|c|c|}
\hline Variable & $\begin{array}{c}\text { VSR group } \\
(\mathbf{n = 6 3})\end{array}$ & $\begin{array}{c}\text { non-VSR group } \\
(\mathbf{n = 1 8 9})\end{array}$ & P value \\
\hline SBP (mmHg, $\bar{X} \pm$ S) & $106.37 \pm 16.12$ & $127.22 \pm 19.50$ & $<0.01$ \\
\hline $\begin{array}{c}\text { DBP }(\mathrm{mmHg}, \mathrm{M}(\mathrm{Q} 1, \\
\text { Q3)] }\end{array}$ & $69.00(62.00,77.00)$ & $79.00(71.50,86.50)$ & $<0.01$ \\
\hline LVEF [\%, M (Q1, Q3)] & $53.00(45.00,57.00)$ & $56.00(48.00,61.00)$ & 0.003 \\
\hline $\begin{array}{c}\text { LVESD [mm, M (Q1, } \\
\text { Q3)] }\end{array}$ & $37.00(34.00,42.00)$ & $35.00(32.00,39.00)$ & 0.02 \\
\hline $\begin{array}{c}\text { NT-proBNP [ng/L, M } \\
(\mathrm{Q} 1, \mathrm{Q} 3)]\end{array}$ & $2960.00(1659.00,7351.00)$ & $786.00(312.50,1973.00)$ & $<0.01$ \\
\hline CK [U/L, M (Q1, Q3)] & $103.00(43.00,230.00)$ & $88.00(55.50,248.00)$ & 0.66 \\
\hline $\begin{array}{c}\text { CK-MB [U/L, M (Q1, } \\
\text { Q3)] }\end{array}$ & $24.00(13.00,37.00)$ & $18.40(12.95,33.00)$ & 0.11 \\
\hline $\begin{array}{c}\text { LDH1 [U/L, M (Q1, } \\
\text { Q3)] }\end{array}$ & $199.00(98.00,374.00)$ & $139.00(62.50,239.50)$ & 0.004 \\
\hline
\end{tabular}

Note: SBP: Systolic Blood Pressure; DBP: Diastolic Blood Pressure; LVEF: Left Ventricular Ejection Fraction; LVESD: Left ventricular end-systolic diameter; NT-proBNP (N-terminal pro-B-type natriuretic peptide): N-terminal B Type natriuretic peptide; CK (Creatine Kinase): creatine kinase; CK-MB: creatine kinase isoenzyme; LDH (lactate dehydrogenase): lactate dehydrogenase; $1 \mathrm{mmHg}=0.133 \mathrm{KPa}$

Table 4: Checks related to myocardial infarction

5. Clinical characteristics of the ventricular septal rupture group: 49 cases (77.8\%) underwent interventional occlusion, 34 cases (69\%) survived 1 year after the operation; 49 cases (78.8\%) with perforation located at the apex of the heart, intervention The timing of treatment was 23.068 .55 days from the diagnosis. A total of 45 cases $(71.4 \%)$ were treated with MCS (mainly IABP). By comparing the survival group and the death group, it was found that LVEF, glycosylated hemoglobin, CK, CK-MB, LDH1, and postoperative LDH1 were significantly different $(\mathrm{P}<0.01$ or 0.05$)$, and the difference was statistically significant (Table 5$)$. Incorporating statistically different indicators in the univariate analysis into the multivariate logistic regression analysis showed (Table 6): The level of glycosylated hemoglobin is related to the mortality of myocardial infarction combined with ventricular septal rupture $(\mathrm{P}<0.01$ or 0.05$)$

\begin{tabular}{|c|c|c|c|}
\hline Variable & Survival group & Death group & P value \\
\hline LVEF $(\%, \bar{X} \pm S)$ & $53.03 \pm 7.17$ & $48.81 \pm 8.75$ & 0.04 \\
\hline HbA1c [\%, M (Q1, Q3)] & $6.10(5.53,7.53)$ & $7.43(6.11,9.37)$ & 0.009 \\
\hline CK [U/L, M (Q1, Q3)] & $72.00(37.00,189.5)$ & $139.00(65.50,713.25)$ & 0.009 \\
\hline CK-MB [U/L, M (Q1, Q3)] & $18.00(11.00,26.00)$ & $34.50(19.85,78.95)$ & 0.003 \\
\hline LDH1 [U/L, M (Q1, Q3)] & $182.00(78.00,268.00)$ & $207.50(128.50,682.00)$ & 0.02 \\
\hline $\begin{array}{c}\text { postoperative LDH1 [U/L, } \\
\text { M (Q1, Q3)] }\end{array}$ & $127.00(68.00,291.00)$ & $280.00(151.50,450.00)$ & 0.02 \\
\hline
\end{tabular}

Table 5: Comparison of data between survival group and death group after ventricular septal rupture treatment 


\begin{tabular}{|c|c|c|c|c|c|c|}
\hline Variable & $\boldsymbol{B}$ & $\boldsymbol{S E}$ & Wald & $\boldsymbol{P}$ & OR & 95\% $\boldsymbol{C I}$ \\
\hline LVEF & -0.06 & 0.07 & 0.74 & 0.39 & 0.94 & $0.83-1.08$ \\
\hline LVESD & 0.05 & 0.08 & 0.5 & 0.55 & 1.05 & $0.90-1.23$ \\
\hline HbA1c & 0.51 & 0.24 & 4.47 & 0.03 & 1.66 & $1.04-2.95$ \\
\hline CK & 0.001 & 0.003 & 0.21 & 0.65 & 1.00 & $0.99-1.00$ \\
\hline CK-MB & 0.02 & 0.02 & 0.74 & 0.39 & 1.02 & $0.96-1.07$ \\
\hline LDH1 & -0.004 & 0.003 & 1.40 & 0.24 & 0.996 & $0.98-1.03$ \\
\hline postoperative LDH1 & 0.002 & 0.002 & 1.79 & 0.18 & 1.002 & $0.99-1.00$ \\
\hline
\end{tabular}

Table 6: Multivariate logistic regression analysis of death group one year after ventricular septal rupture

6. Analysis of related factors of ventricular septal rupture after myocardial infarction (Table 7): According to the above research results, the two groups have significant statistical differences in indicators using multivariate logistic regression analysis. It is found that age $>60$ years old, gender, diseased blood vessels, Albumin $<35 \mathrm{~g} / \mathrm{L}, \mathrm{SBP}<90 \mathrm{mmHg}, \mathrm{LVEF}<40 \%$, LVESD is related to ventricular septal rupture after myocardial infarction $(\mathrm{P}<0.01$ or 0.05$)$.

\begin{tabular}{|c|c|c|c|c|c|c|}
\hline Variable & B & SE & Wald & P & OR & 95\%CI \\
\hline Age $>60$ & -2.13 & 0.50 & 17.78 & $<0.01$ & 0.12 & $0.04-0.32$ \\
\hline Gender & -1.91 & 0.64 & 8.96 & 0.003 & 0.15 & $0.04-0.52$ \\
\hline $\begin{array}{c}\text { Coronary artery } \\
\text { disease }\end{array}$ & 0.51 & 0.21 & 5.83 & 0.02 & 1.66 & $1.10-2.51$ \\
\hline Albumin $<35 \mathrm{~g} / 1$ & -2.10 & 0.51 & 16.99 & $<0.01$ & 0.12 & $0.05-0.33$ \\
\hline SBP $<90 \mathrm{mmHg}$ & -3.08 & 1.06 & 8.40 & 0.004 & 0.05 & $0.006-0.37$ \\
\hline LVEF $<40 \%$ & 1.94 & 0.90 & 4.65 & 0.03 & 6.95 & $1.19-40.46$ \\
\hline LVESD & -0.10 & 0.04 & 6.46 & 0.01 & 0.91 & $0.84-0.98$ \\
\hline
\end{tabular}

Table 7: Multivariate logistic regression analysis of related factors of ventricular septal rupture

\section{Discussion}

Acute myocardial infarction combined with ventricular septal rupture is a serious complication. The patient will have a high oxygen content of blood shunting from the left ventricle to the right ventricle, which manifests as circulatory failure and extremely high mortality [14]. Therefore, for patients with acute myocardial infarction, it is particularly important to detect abnormalities from related examinations and intervene in advance to prevent the occurrence of ventricular septal rupture. This study shows that the admission hemoglobin, albumin, and serum chloride levels of patients with myocardial infarction combined with ventricular septal rupture are different from those of the control group, and there are few reports in the previous literature.

Smoking and heavy drinking are both risk factors for acute myocardial infarction. In smoking patients, the release of prostacyclin is reduced, causing platelets to easily adhere to the arterial wall. At the same time, the nicotine contained in tobacco acts on the coronary arteries and myocardium, causing arterial spasm and myocardial damage. Coagulation and fibrinolysis are important determinants of acute myocardial infarction. After heavy drinking, the plasma plasminogen activator inhibitor-1 (PAI-1) antigen level and PAI-1 activity increased, and the tissue-type plasminogen activator (t-PA) activity decreased. In people who did not drink alcohol, the circadian rhythm of fibrinolysis was enhanced at night and lowest in morning activity. Subjects who drink a lot of alcohol lose the circadian rhythm of fibrinolysis, which leads to the occurrence and development of atherosclerosis. Studies have confirmed that the threshold for significant inhibition of fibrinolysis is equivalent to 20-40 grams of alcohol. In view of the low proportion of smoking and drinking in this study, there is a contradiction with previous studies, firstly because women in the case group accounted 
for the majority; secondly, relative to the control group. Therefore, smoking and drinking are still important risk factors for acute myocardial infarction, leading to vascular endothelial damage, promoting plaque formation, leading to no-reflow and aggravating myocardial damage.

This study shows that the serum total protein and albumin levels of patients with ventricular septal perforation after myocardial infarction are lower than those of the control group, and the decrease of serum total protein is a risk factor for ventricular septal perforation after myocardial infarction. Albumin has important physiological functions in the body and is the main determinant of maintaining plasma colloidal osmotic pressure. It also participates in the transport of large amounts of substances and participates in acute and chronic inflammatory reactions [15]. Previous literature has shown that hypoproteinemia is associated with poor postoperative myocardial reperfusion and low ejection fraction. In the early hospitalization of patients with hypoproteinemia, infarction-related complications (mortality, heart failure, severe arrhythmia) and surgery-related complications (massive hemorrhage, contrast nephropathy) are more common. In addition, long-term follow-up of such patients found that hypoproteinemia was associated with higher cardiogenic and non-cardiogenic mortality, advanced heart failure, stroke, and reinfarction. Hypoproteinemia at admission is an independent predictor of increased long-term mortality and advanced heart failure [16,17]. The possible explanations are as follows. 1. Hypoproteinemia causes water and sodium retention and blood dilution, thereby increasing hemodynamic instability and aggravating the heart load. 2. Serum albumin is related to the transport of inflammatory mediators, and inflammation may consume serum albumin levels. In addition, the reduction of serum albumin may be related to the severity of vascular endothelial disease, leading to the occurrence of no-reflow, thereby reducing the perfusion of the epicardium and tissues. There are also reports that hypoalbuminemia can lead to myocardial edema and aggravate the decrease in ejection fraction. Therefore, in summary, when patients with acute myocardial infarction also have hypoproteinemia, under the action of inflammatory factors, the patient's vascular endothelial damage will be accelerated, leading to further accumulation of platelets and thrombi, and the patient's heart volume load will further increase. Increase the work of the heart, which leads to the occurrence of ventricular septal perforation.

Chloride ion is the most important anion in plasma and tissue fluid, accounting for about one-third of plasma tension and twothirds of all negative ions in plasma. Although chloride ions are important, they have received little attention in cardiovascular diseases. This study proved that patients with acute myocardial infarction with hypochloremia are more prone to ventricular septal perforation. In patients with acute myocardial infarction and heart failure, hyponatremia is a recognized, powerful and independent predictor of short-term and long-term morbidity and mortality [18-21]. Previous studies have shown that serum chloride ion levels have been found to be independent and negatively correlated with mortality [22,23]. To a certain extent, the volume and $\mathrm{pH}$ of cardiomyocytes are regulated by chloride-assisted transporters. Abnormalities in chloride channels and cotransporters can cause arrhythmias and impair contractility. A study found that compared with patients with normal chloride levels, patients with heart failure and hypochloremia had higher excretion of serum bicarbonate and some chlorides ${ }^{[24]}$, leading to metabolic alkalosis in patients. Most patients with acute myocardial infarction combined with ventricular septal perforation have heart failure, resulting in insufficient renal perfusion. The use of large amounts of diuretics will activate the renin-angiotensin-aldosterone (RASS) system, which will further lead to the further excretion of chloride ions. Maintain electrical neutrality. Therefore, compared with the control group, when the patient has hypochloremia, it will lead to excessive metabolism and volume load in the body, thereby accelerating the occurrence of ventricular septal perforation after acute myocardial infarction. For such problems, there is currently no exact research mechanism to confirm the relevance. therefore. In clinical work, we should pay attention to the role and influence of chloride ions, and analyze the interactions with other ions and the potential causes of unbalanced adjustments, which may help clinicians improve the prediction of results and the formulation of treatment strategies.

Patients with ventricular septal perforation after acute myocardial infarction may be related to diseased blood vessels. Compared with the control group, in this study, patients with ventricular septal perforation are mainly the left anterior descending artery, and the location of the perforation is mostly in the apex of the heart. It may be a risk factor for ventricular septal perforation after acute myocardial infarction. The previous literature also supports this view $[25,26]$. Research also suggests that advanced age and women are also risk factors for ventricular septal perforation after acute myocardial infarction $[27,28]$. 
In addition, the transitional inflammatory response and the structural changes of the extracellular matrix (ECM) are also considered to be the cause of heart rupture after myocardial infarction [29,30]. Among them, matrix metalloproteinases play an important role [31]. Matrix metalloproteinases (MMP) is a family of zinc-containing endoproteases mainly released by white blood cells. They are mainly secreted by inflammation and tumor cells as proenzymes. MMP-9 is highly expressed in vulnerable areas of atherosclerotic plaques, reflecting The vulnerability of the plaque [32,33]. It plays a key role in the process of degrading the extracellular matrix of the heart [34]. Specifically, MMP-9 not only participates in the destruction of ECM, but also participates in the infiltration of white blood cells in the heart, cytokine activation and angiogenesis. Therefore, inflammation not only plays an important role in the pathophysiology of the disease, but also affects the progression of the disease, and may be an important intervention target in the primary and secondary prevention of the disease.

The treatment for such patients mainly includes reduction of afterload, use of diuretics, and usually the use of external mechanical assist devices and oxygenated inotropic drugs. Transcatheter intervention can immediately reduce the shunt to prevent the deterioration of hemodynamics. Therefore, transcatheter closure has become a valuable alternative to surgical repair, with a high surgical success rate [35]. This study shows that after the occurrence of ventricular septal perforation, the overall survival rate of active interventional therapy is higher, reaching $68.9 \%$, which is similar to other clinical experimental research data [6,36]. However, a large number of studies have observed heterogeneity regarding the timing of transcatheter closure [11-37]. The current guidelines of the European Society of Cardiology (ESC) and the American College of Cardiology Foundation/American Heart Association (ACCF/AHA) suggest that VSR closure treatment after AMI is urgently recommended regardless of the hemodynamic status [38,39]. However, in clinical practice, the timing and perioperative treatment of VSR closure after AMI are still controversial. In previous studies, most percutaneous catheter closures were performed in the subacute and chronic phases ( $\geq 2$ weeks) after the first detection of AMI-VSR. According to reports, the mortality rate of delayed occlusion (AMI-VSR $\geq 2$ weeks after detection) is 6.1-10.0\%, while the mortality rate of early occlusion ( $<2$ weeks after the occurrence of AMI-VSR) is as high as $66 \%$. This may be because pre-medical treatment or mechanical support (such as IABP) can stabilize the patient's hemodynamic status and prolong the operation time so that the myocardium has enough time to repair and form myocardial scar tissue. Similar to most studies, the timing of interventional therapy in this study was 22.309 .00 days, the success rate of interventional closure was higher, and the overall prognosis of patients was better. Therefore, for such patients, if the patient's condition permits, it is recommended to extend the occlusion.

\section{Limitations}

This study is a single-center small-sample study, and because most of the patients in this center are diagnosed and transferred, the study is biased. Due to the poor prognosis of patients and fewer surviving patients, long-term follow-up of surviving patients was not carried out. In addition, this study also lacks histopathological evidence, as well as basic experimental research.

\section{Conclusions}

In summary, after myocardial infarction combined with ventricular septal rupture is a rare but extremely fatal complication. Among them, acute anterior wall myocardial infarction has a higher probability of this complication. For the elderly, women, low blood pressure, low hemoglobin, and low blood pressure Patients with albuminemia, hypochloremia, and left ventricular insufficiency should pay attention to preventing the occurrence of ventricular septal rupture and reducing the mortality of patients with myocardial infarction. 


\section{References}

1. Crenshaw B, Granger C, Birnbaum Y (2000) Risk factors, angiographic patterns, and outcomes in patients with ventricular septal defect complicating acute myocardial infarction. GUSTO-I (Global Utilization of Streptokinase and TPA for Occluded Coronary Arteries) Trial Investigators. Circulation 101: 27-32.

2. Parachuri V, Tripathy A, Gaikwad N (2019) Modified Infarct Exclusion Technique for Repair of Postinfarction Ventricular Septal Rupture. The Annals of Thoracic Surgery 107: e219-e221.

3. Dogra N, Puri G, Thingnam S (2019) Early thrombolysis is associated with decreased operative mortality in postinfarction ventricular septal rupture. Indian Heart Journal 71: 224-8.

4. Elbadawi A, Elgendy I, Mahmoud K (2019) Temporal Trends and Outcomes of Mechanical Complications in Patients with Acute Myocardial Infarction. JACC Cardiovascular Interventions 12: 1825-36.

5. French J, Hellkamp A, Armstrong P (2010) Mechanical complications after percutaneous coronary intervention in ST-elevation myocardial infarction (from APEX-AMI). The American Journal of Cardiology 105: 59-63.

6. Moreyra A, Huang M, Wilson A (2010) Trends in incidence and mortality rates of ventricular septal rupture during acute myocardial infarction. The American Journal of Cardiology 106: 1095-100.

7. Yamanaka T, Fukatsu T, Ichinohe Y (2017) Spontaneous closure of ventricular septal perforation following percutaneous coronary intervention for acute myocardial infarction. BMJ Case Reports 2017.

8. Vargas-Barron J, Molina-Carrion M, Romero-Cárdenas A (2005) Risk factors, echocardiographic patterns, and outcomes in patients with acute ventricular septal rupture during myocardial infarction. The American Journal of Cardiology 95: 1153-8.

9. Wang C, Zhang C, Liu L (2017) Macrophage-Derived mir-155-Containing Exosomes Suppress Fibroblast Proliferation and Promote Fibroblast Inflammation during Cardiac Injury. Molecular therapy: the journal of the American Society of Gene Therapy 25: 192-204.

10. El-Aziz TAA, Mohamed RH (2017) Matrix metalloproteinase -9 polymorphism and outcome after acute myocardial infarction. International Journal of Cardiology 227: 524-8.

11. Furui M, Yoshida T, Kakii B (2018) Strategy of delayed surgery for ventricular septal perforation after acute myocardial infarction. Journal of Cardiology 71: 488-93.

12. Thygesen K, Alpert JS, Jaffe AS (72) Fourth Universal Definition of Myocardial Infarction (2018).Journal of the American College of Cardiology 72: 2231-64.

13. Jones B, Kapadia S, Smedira N (2014) Ventricular septal rupture complicating acute myocardial infarction: a contemporary review. European Heart Journal 35: 2060-8.

14. Bajaj A, Sethi A, Rathor P (2015) Acute Complications of Myocardial Infarction in the Current Era: Diagnosis and Management. Journal of Investigative Medicine: the official publication of the American Federation for Clinical Research 63: 844-55. 
15. Eckart A, Struja T, Kutz A (2020) Relationship of Nutritional Status, Inflammation, and Serum Albumin Levels During Acute Illness: A Prospective Study. The American Journal of Medicine 133: 713-722.e717.

16. Oduncu V, Erkol A, Karabay C (2013) The prognostic value of serum albumin levels on admission in patients with acute ST-segment elevation myocardial infarction undergoing a primary percutaneous coronary intervention. Coronary Artery Disease 24: $88-94$.

17. Polat N, Oylumlu M, Isik M (2020) Prognostic Significance of Serum Albumin in Patients With Acute Coronary Syndrome. Angiology 71: 903-8.

18. Goldberg A, Hammerman H, Petcherski S (2006) Hyponatremia and long-term mortality in survivors of acute ST-elevation myocardial infarction. Arch Intern Med 166: 781-6.

19. Goldberg A, Hammerman H, Petcherski S (2004) Prognostic importance of hyponatremia in acute ST-elevation myocardial infarction. The American Journal of Medicine 117: 242-8.

20. Klein L, O'Connor CM, Leimberger JD (2005) Lower serum sodium is associated with increased short-term mortality in hospitalized patients with worsening heart failure: results from the Outcomes of a Prospective Trial of Intravenous Milrinone for Exacerbations of Chronic Heart Failure (OPTIME-CHF) study. Circulation 111: 2454-60.

21. Gheorghiade M, Abraham WT, Albert NM (2007) Relationship between admission serum sodium concentration and clinical outcomes in patients hospitalized for heart failure: an analysis from the OPTIMIZE-HF registry. European Heart Journal 28: 980-8.

22. Grodin J, Simon J, Hachamovitch R (2015) Prognostic Role of Serum Chloride Levels in Acute Decompensated Heart Failure. Journal of the American College of Cardiology 66: 659-66.

23. Grodin JL, Verbrugge FH, Ellis SG (2016) Importance of Abnormal Chloride Homeostasis in Stable Chronic Heart Failure. Circulation Heart Failure 9: e002453.

24. Hanberg J, Rao V, Ter Maaten J (2016) Hypochloremia and Diuretic Resistance in Heart Failure: Mechanistic Insights. Circulation Heart Failure 9.

25. Nozoe M, Sakamoto T, Taguchi E (2014) Clinical manifestation of early phase left ventricular rupture complicating acute myocardial infarction in the primary PCI era. Journal of Cardiology 63: 14-8.

26. Ptaszynska-Kopczynska K, Sobolewska D, Kożuch M (2011) Efficacy of invasive treatment and the occurrence of cardiac rupture in acute ST-elevation myocardial infarction. Kardiol Pol 69: 795-800.

27. López-Sendón J, Gurfinkel E, Lopez De Sa E (2010) Factors related to heart rupture in acute coronary syndromes in the Global Registry of Acute Coronary Events. European Heart Journal 31: 1449-56.

28. Qian G, Liu H, Wang J (2013) Risk of cardiac rupture after acute myocardial infarction is related to a risk of hemorrhage. Journal of Zhejiang University Science B 14: 736-42.

29. Anzai T (2013) Post-infarction inflammation and left ventricular remodeling: a double-edged sword. Circulation Journal: Official Journal of the Japanese Circulation Society 77: 580-7. 
30. Fang L, Gao X, Moore X (2007) Differences in inflammation, MMP activation and collagen damage account for gender difference in murine cardiac rupture following myocardial infarction. Journal of Molecular and Cellular Cardiology 43: 535-44.

31. Kameda K, Matsunaga T, Abe N (2006) Increased pericardial fluid level of matrix metalloproteinase-9 activity in patients with acute myocardial infarction: possible role in the development of cardiac rupture. Circulation Journal: Official Journal of the Japanese Circulation Society 70: 673-8.

32. Uzui H, Harpf A, Liu M (24) Increased expression of membrane type 3-matrix metalloproteinase in human atherosclerotic plaque: role of activated macrophages and inflammatory cytokines. Circulation 106: 3024-30.

33. Muzzio M, Miksztowicz V, Brites F (2009) Metalloproteases 2 and 9, Lp-PLA(2) and lipoprotein profile in coronary patients. Archives of Medical Research 40: 48-53.

34. Creemers E, Cleutjens J, Smits J (2001) Matrix metalloproteinase inhibition after myocardial infarction: a new approach to prevent heart failure? Circulation Research 89: 201-10.

35. Schlotter F, de waha S, Eitel I (2016) Interventional post-myocardial infarction ventricular septal defect closure: a systematic review of current evidence. EuroIntervention: journal of EuroPCR in collaboration with the Working Group on Interventional Cardiology of the European Society of Cardiology 12: 94-102.

36. Premchand R, Garipalli R, Padmanabhan T (2017) Percutaneous closure of post-myocardial infarction ventricular septal rupture - A single centre experience. Indian heart journal 2017: S24-S27.

37. Huang S, Wang C (2015) Risk factors and outcome analysis after surgical management of ventricular septal rupture complicating acute myocardial infarction: a retrospective analysis. Journal of Cardiothoracic Surgery 10: 66.

38. Ibanez B, James S, Agewall S (2017) ESC Guidelines for the management of acute myocardial infarction in patients presenting with ST-segment elevation: The Task Force for the management of acute myocardial infarction in patients presenting with ST-segment elevation of the European Society of Cardiology (ESC). European Heart Journal 39: 119-77.

39. O'gara P, Kushner F, Ascheim D (2013) ACCF/AHA guideline for the management of ST-elevation myocardial infarction: a report of the American College of Cardiology Foundation/American Heart Association Task Force on Practice Guidelines. Circulation 127: e362-425. 